\title{
mTOR-Mediated Activation of p70 S6K Induces Differentiation of Pluripotent Human Embryonic Stem Cells
}

\author{
Charles A. Easley, IV,, Ahmi Ben-Yehudah,,2 Carrie J. Redinger, Stacie L. Oliver, ${ }^{1,2}$ Sandra T. Varum,,2 \\ Vonya M. Eisinger, Diane L. Carlisle,, ${ }^{1,2}$ Peter J. Donovan, ${ }^{3}$ and Gerald P. Schatten ${ }^{1,2}$
}

\begin{abstract}
Deciding to exit pluripotency and undergo differentiation is of singular importance for pluripotent cells, including embryonic stem cells (ESCs). The molecular mechanisms for these decisions to differentiate, as well as reversing those decisions during induced pluripotency (iPS), have focused largely on transcriptomic controls. Here, we explore the role of translational control for the maintenance of pluripotency and the decisions to differentiate. Global protein translation is significantly reduced in hESCs compared to their differentiated progeny. Furthermore, p70 S6K activation is restricted in hESC s compared to differentiated fibroblast-like cells. Disruption of p70 S6K-mediated translation by rapamycin or siRNA knockdown in undifferentiated hESCs does not alter cell viability or expression of the pluripotency markers Oct4 and Nanog. However, expression of constitutively active p70 S6K, but not wild-type p70 S6K, induces differentiation. Additionally, hESCs exhibit high levels of the mTORC1/p70 S6K inhibitory complex TSC1/TSC2 and preferentially express more rapamycin insensitive mTORC2 compared to differentiated cells. siRNA-mediated knockdown of both TSC2 and Rictor elevates p70 S6K activation and induces differentiation of hESCs. These results suggest that hESCs tightly regulate mTORC1/p70 S6K-mediated protein translation to maintain a pluripotent state as well as implicate a novel role for protein synthesis as a driving force behind hESC differentiation.
\end{abstract}

\section{Introduction}

$\mathbf{E}_{\mathrm{p}}^{\mathrm{s}}$ MBRYONIC STEM CELls (ESC) provide great clinical promise for cell-based therapies because these cells can differentiate into all adult cell lineages. Although significant strides have been made in understanding the transcriptional factors regulating differentiation of ESCs, protein translation has not yet been investigated as rigorously. Microarray analysis has shown several genes vital for pluripotency, including Oct4, Sox2, and Nanog, as well as a large number of genes responsible for differentiation, such as Gata6, Hand1, and Hoxb1 (Dvash et al., 2004; Gunji et al., 2004; Ivanova et al., 2002, 2006; Lee et al., 2006; Pritsker et al., 2006; RamalhoSantos et al., 2002; Walker et al., 2007). Yet, microarray data do not correlate with protein expression differences between ESC and differentiated cells (Chang and Stanford, 2008). Recent studies have explored translational control as a means of bridging the information divide between microarray analysis and proteomic profiling (Chang and Stanford, 2008; Sampath et al., 2008). These results have shown that global translation rates are decreased in mESCs compared to differentiated cells obtained from mouse ESC embryoid body formation (Sampath et al., 2008). Also, although global translation rates have been evaluated, the signaling cascades that regulate protein translation have not yet been investigated in either mESCs or hESCs.

mTOR (mammalian target of rapamycin) is a member of the PIKK (phosphoinositide 3-kinase-related kinase) family with homologs in all eukaryotes (Dennis et al., 2001; Gingras et al., 2001a, 2001b; Raught et al., 2001; Sabers et al., 1995; Wiederrecht et al., 1995). Primarily, mTOR activity has been linked with cell growth, proliferation, survival, protein translation, and other cellular metabolic processes (Dennis et al., 2001; Gingras et al., 2001a, 2001b; Raught et al., 2001;

\footnotetext{
${ }^{1}$ Pittsburgh Development Center; Magee-Womens Research Institute and Foundation, Pittsburgh, Pennsylvania

${ }^{2}$ Division of Developmental and Regenerative Medicine, Departments of Obstetrics, Gynecology, and Reproductive Sciences; University of Pittsburgh, Pennsyvlania.

${ }^{3}$ Sue and Bill Gross Stem Cell Research Program, Department of Developmental and Cell Biology, University of California Irvine, Irvine, California.
} 
Sabers et al., 1995; Wiederrecht et al., 1995). Two functionally distinct mTOR complexes exist in mammalian cells: mTORC1 (mTOR complex 1), which contains mTOR, Raptor, and LST8; and mTORC2, which contains Rictor, LST8, and Sin1 (Loewith et al., 2002). Among its many functions, mTORC1 promotes protein translation through activation of p70 S6K and inhibition of 4E-BP1 (eukaryotic initiation factor $4 \mathrm{E}$ binding protein) (Fang et al., 2001). mTORC2 regulation and function remains largely unknown, although this complex has been linked to cytoskeletal rearrangements and cell survival through Akt (Jacinto et al., 2004). mTORC2 is also rapamycin-insensitive.

p70 S6K, a serine/threonine kinase, is a major regulator of translation and is activated through phosphorylation events within the catalytic, linker, and pseudosubstrate domains by the mTORC1 pathway (Alessi et al., 1998; Pullen and Thomas, 1997; Pullen et al., 1998; Weng et al., 1998). Once activated, p70 S6K phosphorylates the S6 protein of the 40S ribosomal subunit and the translation initiation factor, eIF4B (Gingras et al., 2001a; Pullen and Thomas, 1997) to promote translation. Due to its major function in regulating translation, p70 S6K is required for cell growth and G1 cell cycle progression (Dufner and Thomas, 1999; Pullen and Thomas, 1997). Although the role of p70 S6K in regulating translation in differentiated cells has been well characterized, its function in hESCs has not been rigorously examined.

In this study, we show that mTORC1 activity is tightly regulated in human ESCs (hESCs), and thus p70 S6K activation is decreased in hESCs compared to their differentiated counterparts. Surprisingly, p70 S6K-mediated translation is dispensable for the maintenance of pluripotency in hESCs. In contrast, elevation in p70 S6K activation, through either expression of a constitutively active mutant p70 S6K, or increased mTORC1 activity causes differentiation of hESCs. Together, these results describe a novel role for $\mathrm{mTORC} 1 / \mathrm{p} 70$ $\mathrm{S} 6 \mathrm{~K}$ in regulating differentiation of pluripotent stem cells and highlight the importance of protein translation as another modulator of differentiation.

\section{Materials and Methods}

\section{Cell culture}

H7 hESCs (WiCell, Madison, WI, USA) were cultured at $37^{\circ} \mathrm{C}, 5 \% \mathrm{CO}_{2}$ on mitomycin $\mathrm{C}$-treated mouse embryonic fibroblasts (MEF; Chemicon, Millipore Corporation, Billerica, MA, USA) in $80 \%$ Knockout Medium (Invitrogen, Carlsbad, CA, USA); 20\% Knockout Serum Replacement (Invitrogen); $1 \mathrm{mM}$ L-glutamine (Invitrogen); $0.1 \mathrm{mM}$ Nonessential Amino Acids (Invitrogen); 1\% Penicillin/Streptomycin (Invitrogen); $4 \mathrm{ng} / \mathrm{mL}$ of bFGF (Invitrogen). H7 hESC were passaged mechanically every 6-7 days as need using the StemPro EZPassage tool (Invitrogen). For feeder-free conditions, we used the mTeSR ${ }^{\mathrm{TM}} 1$ system (StemCell Technologies, Vancouver, BC Canada) and cultured H7 hESCs on hESCqualified BD Matrigel ${ }^{\mathrm{TM}}$ matrix (BD Biosciences, San Jose, CA, USA). We passaged hESCs grown in feeder-free conditions every 5-6 days using dispase as described (Ludwig et al., 2006a, 2006b). H7 differentiated fibroblasts derived from teratomas were cultured at $37^{\circ} \mathrm{C}, 5 \% \mathrm{CO}_{2}$ in Dulbecco's Modified Eagle Medium (DMEM, Invitrogen) containing $10 \%$ heat-inactivated fetal bovine serum (FBS, Invitrogen), $1 \mathrm{mM}$ L-glutamine (Invitrogen), 1\% Penicillin/Streptomycin
(Invitrogen), and $0.1 \mathrm{mM}$ Nonessential Amino Acids (Invitrogen). Cells were chemically passaged every 3 or 4 days, as needed, using TrypLE (Invitrogen).

\section{Teratoma fibroblast isolation}

Teratomas derived from H7 hESC were surgically removed from nonobese diabetic/severe combined immunodeficienty (NOD/SCID) mice (Navara et al., 2007a). Excised teratomas were then placed in 60-mm tissue culture dishes (Nunc, Thermo Fisher Scientific, Rochester, NY, USA) and washed three times with phosphate-buffered saline solution (PBS, Invitrogen) containing 1\% Penicillin/Streptomycin. Washed teratomas were then minced with dissecting scissors in $2 \mathrm{~mL}$ of TrypLE and incubated at $37^{\circ} \mathrm{C}$ for $5 \mathrm{~min}$. Minced samples were then transferred to a $0.1 \%$ gelatin-coated T-25 tissue culture flask (Corning, Lowell, MA, USA) and cultured in fibroblast medium noted above. After 5-7 days, minced teratoma sections were removed and fibroblasts were passaged as mentioned above. Teratoma-derived fibroblasts were cultured more than five passages before use in this study.

\section{Transmission electron microscopy (TEM)}

hESCs were cultured in a feeder-free condition and differentiated fibroblasts were cultured as previously described. Cells were rinsed briefly with PBS solution and fixed in $2.5 \%$ glutaraldehyde (Sigma, St. Louis, MO, USA) for $1 \mathrm{~h}$ at room temperature. After fixation, cells were incubated in PBS and stored at $4{ }^{\circ} \mathrm{C}$ prior to processing. For processing, samples were fixed with $1 \%$ osmium tetroxide for $1 \mathrm{~h}$ at $4{ }^{\circ} \mathrm{C}$. Samples were then washed with PBS for $10 \mathrm{~min}$. Samples were then dehydrated using a graded ethanol series. After dehydration, samples were incubated overnight in $100 \%$ Epon solution at $37^{\circ} \mathrm{C}$, and 2 days later at $60^{\circ} \mathrm{C}$. Sections $(70 \mathrm{~nm})$ were then imaged using a 1011 CX Transmission Electron Microscope (JEOL, Tokyo, Japan).

\section{Western blot analysis}

Sample preparation and Western blot analysis were performed as previously described (Easley et al., 2008). Immunoblots were probed with the indicated primary antibody, with an appropriate HRP-conjugated secondary and developed using the Amersham ECL Advance Western Blotting Detection Kit (GE Healthcare, Piscataway, NJ, USA).

\section{Global translation detection}

H7 hESC and H7 Diff were cultured as described above in the presence of $10 \mu \mathrm{Ci} / \mathrm{mL}\left[{ }^{35} \mathrm{~S}\right]$ methionine (Perkin-Elmer, Waltham, MA, USA). Cells were harvested as previously described (Easley et al., 2008) and subjected to SDS-PAGE. Radioactive gels were then transferred to nitrocellulose and assessed by autoradiography.

\section{Amaxa nucleofection}

H7 hESC were nucleofected as previously described (Hohenstein et al., 2008) using the Amaxa Nucleofector II system and the Human Stem Cell Nucleofector Kit 2 (Lonza, Gaithersburg, MD, USA). For p70 S6K and GFP construct nucleofection, $4 \mu \mathrm{g}$ of total plasmid DNA were used. For siRNA studies, $0.1 \mathrm{~nm}$ of indicated siRNA were used. 


\section{Reagents}

The pmaxGFP construct was provided within the Amaxa Human Stem Cell Nucleofector Kit 2 (Lonza). The p70 S6K constructs were generously donated to Addgene by Dr. John Blenis, Addgene catalog number 8989 (p70 S6K CON) and 8984 (p70 S6K WT). The Oct4 antibody was from Santa Cruz (Santa Cruz, CA, USA). The Nanog antibody was from Kamiya Biomedical Company (Seattle, WA, USA). The Actin, p70 S6K, phospho-S389 p70 S6K, mTOR, eIF4B, and phospho-S422 eIF4B, eIF2B $\varepsilon$, TSC1, TSC2, Raptor, and Rictor antibodies and TSC2 siRNA were all from Cell Signaling (Danvers, MA, USA). Fluorescent and HRP-conjugated secondary antibodies were from Jackson ImmunoResearch Labs (West Grove, PA, USA). Rapamycin, puromycin and emetine were from EMDB Biosciences (Gibbstown, NJ, USA). Scramble low GC-content siRNA, p70 S6K (RPS6KB1) Validated Stealth RNAi DuoPak was from Invitrogen.

\section{Statistical analyses}

Approximately $300 \mathrm{GFP}+$ cells from three separate experiments for each condition were analyzed for Oct4 expression. Percentage plus standard deviation are presented. Statistical significance was determined using the Student's t-test, with $p<0.05$.

\section{Imaging}

Cell fixation was performed as previously described (Navara et al., 2007a). Live or fixed cell imaging was performed on either a Nikon Eclipse TE200-E or Nikon Eclipse $\mathrm{Ti}$ and processed with Nikon NIS Elements software.

\section{Results}

\section{hESCs exhibit lower levels of global protein translation compared to differentiated cells}

To study the translational regulation differences between hESCs and differentiated cells, we derived fibroblasts from a teratoma generated by the injection of the parent human embryonic stem cell line, $\mathrm{H} 7$ (WiCell) into the testes of nude mice for teratoma formation (Navara et al., 2007a, 2007b). After approximately 2-3 months, teratomas were removed, minced, and cultured in DMEM containing 10\% FBS (see Materials and Methods). Once fibroblast-like outgrowth from the teratoma samples was detected, the teratoma sections were removed, and fibroblast-like cells were passaged onto new culture dishes for more than five passages prior to use in this study. H7-derived fibroblast-like cells (referred to as H7 differentiated fibroblasts or H7 Diff) exhibited typical fibroblast morphology and did not grow as colonies in culture like the parent H7 hESC cell line (Fig. 1A). Likewise, these H7 Diff cells do not express pluripotency markers but do possess a normal karyotype (Supplemental Fig. 1; see online supplementary material at www.liebertonline.com).

Previous work in mESCs indicates that pluripotent cells show reduced levels of global translation compared to differentiated cells (Sampath et al., 2008). To analyze whether this result is applicable to hESCs, we examined ER and Golgi morphology in H7 hESCs and their differentiated progeny using TEM. hESCs exhibited immature rough ER and Golgi and fewer polyribosomes compared to their differentiated progeny (Fig. 1B, see arrows). Specifically, the size of Golgi and rough ER organelles were noticeably reduced in hESCs compared to differentiated cells. To further demonstrate that hESCs exhibit decreased protein translation compared to differentiated cells, we cultured H7 hESCs and H7 Diffs in the presence of $\left[{ }^{35} \mathrm{~S}\right]$ methionine for $5 \mathrm{~h}$. Cells were then harvested for total protein and subjected to SDS-PAGE. Equal amounts of total protein were loaded for each condition as determined by Western blot for $\beta$-actin (Fig. 1C). H7 Diffs exhibited an approximately sixfold increase in global translation compared to H7 hESCs as detected by autoradiography (Fig. 1C and D). Thus, like mESCs, differentiation of hESCs is accompanied by a considerable increase in protein synthesis.

\section{p70 S6K signaling is decreased in undifferentiated hESCs compared to their differentiated progeny}

Because p70 S6K is an important regulator of protein translation, we next determined whether expression and/or activation of p70 S6K were dissimilar in H7 Diffs compared to H7 hESCs. Although levels of total p70 S6K were equivalent in the two cell types, H7 Diffs exhibited a significant increase in activated p70 S6K as determined by Western blot analysis for the activation phosphorylation site p-S389 (Fig. 2A and Supplemental Fig. 2A). Furthermore, Western blot analysis revealed that eIF4B, a protein translation initiation factor and a known target of p70 S6K, was increasingly phosphorylated in H7 Diffs compared to H7 hESCs (Fig. 2A). Similarly, phosphorylation of the ribosomal protein S6 was reduced in H7 hESCs compared to their differentiated progeny (Fig. 2B). These results suggest that p70 S6K-mediated protein translation is significantly reduced in pluripotent stem cells compared to their differentiated progeny.

\section{Disruption of p70 S6K-mediated translation does not increase cell death, impair colony formation, or decrease expression of pluripotency markers in $H 7$ hESCS}

Rapamycin, a bacterial macrolide, disrupts mTORC1 activation/signaling by binding to FKBP12 and blocking autophosphorylation on S2481 to impair p70 S6K activation (Holz and Blenis, 2005). To determine whether perturbation of p70 S6K-mediated translation influences cell survival of H7 hESCs and H7 Diffs, we treated cells with $20 \mathrm{nM}$ rapamycin for $72 \mathrm{~h}$, resulting in total deactivation of mTORC1 as determined by Western blot analysis for the autophosphorylation site on mTOR (Supplemental Fig 2C and D). To eliminate off-target effects from rapamycin treatment on hESCs grown on feeders, we also cultured hESCs in the presence of rapamycin in a feeder-free system. Although 72-hour treatment with rapamycin increased cell death in the feeder layer (Supplemental Fig. 2B), no differences in hESC colony viability were observed in a feeder versus feeder-free system (data not shown). Specifically, H7 hESCs exhibited negligible cell death compared to their differentiated progeny, where $\sim 70 \%$ cell death was routinely observed (Fig. 3A).

Because rapamycin treatment did not significantly increase cell death in H7 hESCs, we examined whether inhibition of mTORC1-mediated p70 S6K signaling affected pluripotency. Rapamycin treatments, as performed in this study, have previously been shown to disrupt p70 S6K activation (Kawasome et al., 1998) (Supplemental Fig 2C and D). H7 hESCs were 
A

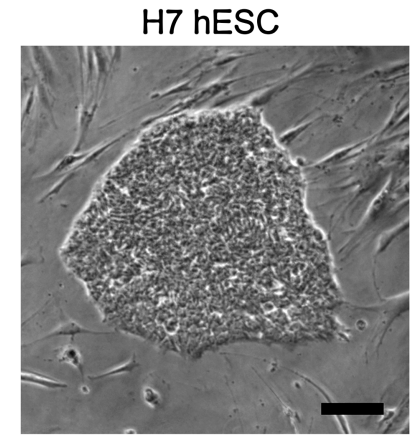

B

FIG. 1. H7 hESCs exhibit decreased protein translation rates compared to differentiated cells. (A) Phase images of H7 hESCs, teratomaderived, and differentiated H7 hESCs (H7 Diffs). Scale bar, $100 \mu \mathrm{m}$. (B) hESCs exhibit immature Golgi and rough ER compared to differentiated cells. TEM images with arrows indicating Golgi and rough ER in H7 hESCs and H7 Diffs. Scale bar, $500 \mathrm{~nm}$. (C) hESCs exhibit decreased rates of global protein translation compared to differentiated cells. Autoradiograph representative of four separate trials showing reduced protein synthesis in cells grown in the presence of $\left[{ }^{35} \mathrm{~S}\right]$ methionine. Western blot analysis for Actin is shown as a loading control.

(D) Graphical analysis of four separate $\left[{ }^{35}\right.$ S]methionine incorporation assays illustrating reduced rates of global protein synthesis in hESCs compared to differentiated cells $(p<0.01)$.

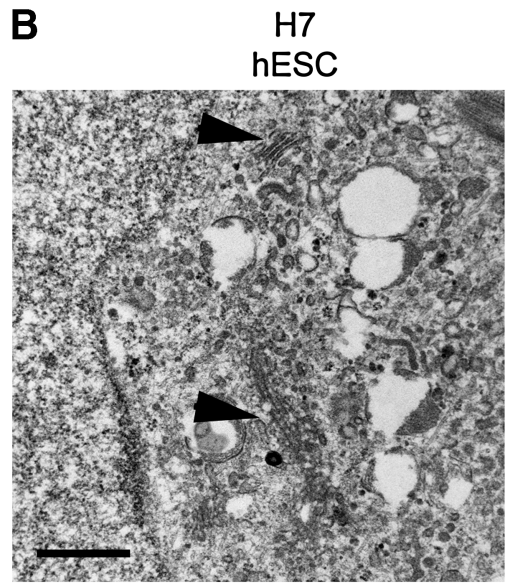

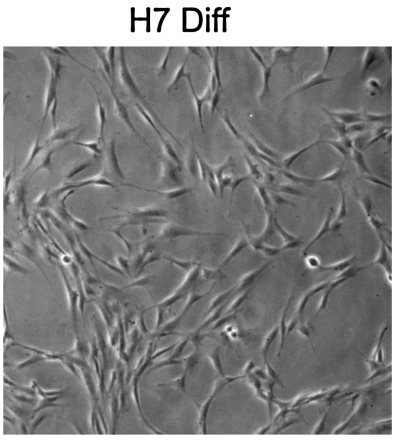
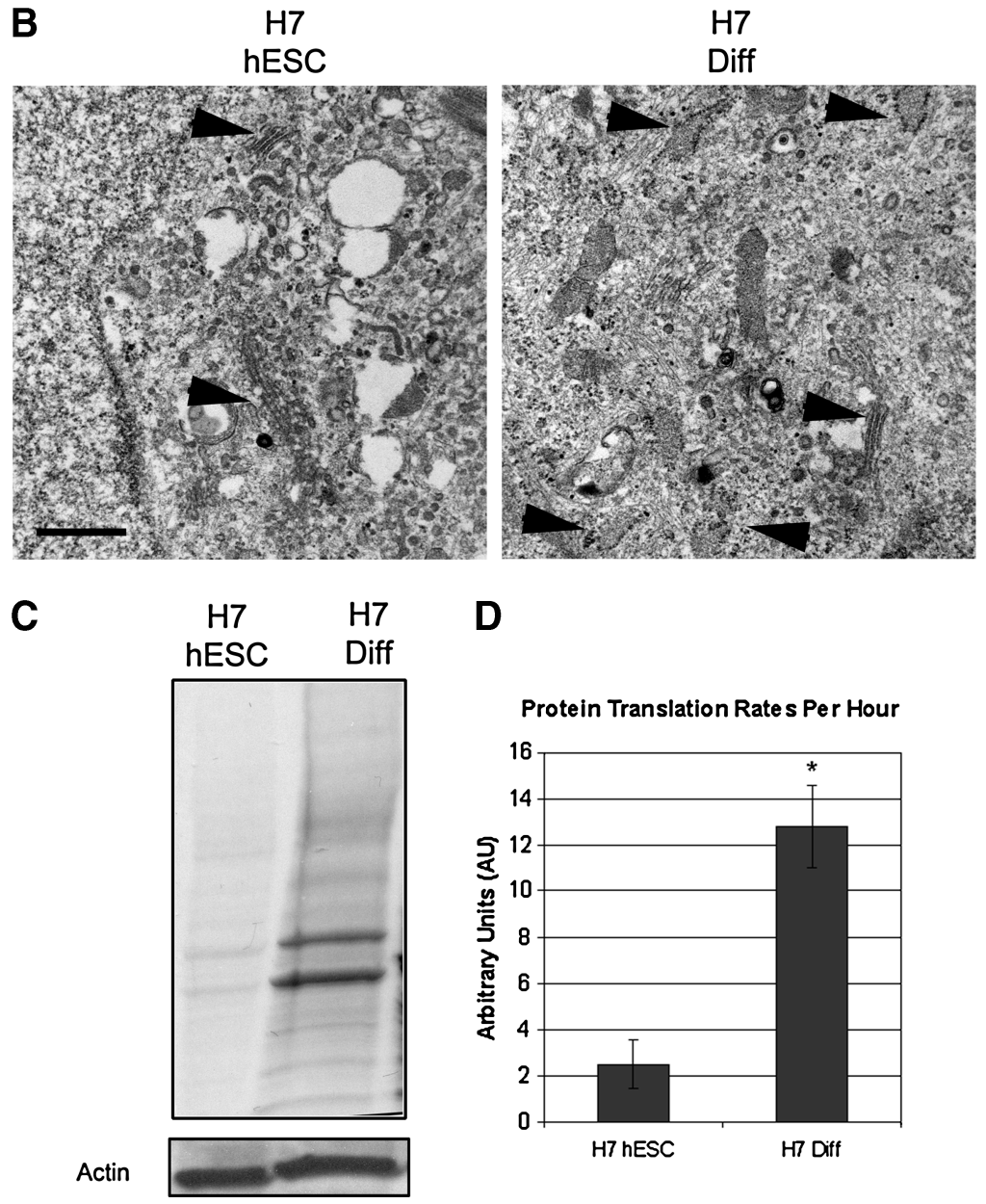

D

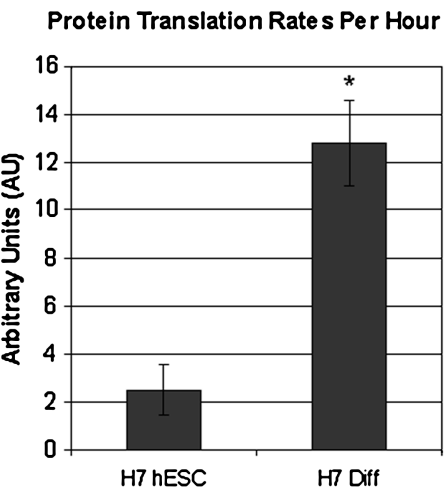

cultured for $72 \mathrm{~h}$ in the presence of $20 \mathrm{nM}$ rapamycin or in the presence of a control vehicle (DMSO) and fixed and probed for Oct4 or Nanog expression. Treatment with rapamycin did not impair stem cell colony formation (Fig. 3B), although we did occasionally observe decreased colony sizes with rapamycin treatment. Rapamycin-treated H7 hESCs also did not demonstrate a loss of Oct4 or Nanog expression (Fig. 3B).

To show that $\mathrm{p} 70 \mathrm{~S} 6 \mathrm{~K}$ is dispensable for the maintenance of pluripotency in hESCs, we selectively knocked down expression of p70 S6K. Using the Amaxa Nucleofector II system (Hohenstein et al., 2008), we nucleofected H7 hESCs with either scramble siRNA or siRNA directed against $\mathrm{p} 70$ S6K. To monitor nucleofection efficiency, we also included a nonspecific fluorescent oligonucleotide. Our nucleofection efficiency was routinely greater than $90 \%$ (data not shown) and indicated a greater than $70 \%$ knockdown in p70 S6K expression (Fig. 3B, Western blot insert). Following nucleofection, H7 hESCs were cultured for 3 days, fixed, and stained for Oct4 or Nanog. Like rapamycin treatment, a knockdown of p70 S6K expression did not impair pluripotent colony formation, Oct4, or Nanog expression in H7 hESCs (Fig. 3B). These results, taken together, suggest that suppression of mTORC1-mediated protein translation does not disrupt pluripotency in H7 hESCs.

\section{Prolonged treatment with rapamycin reduces expression of protein translation initiation factors}

mTORC1-mediated protein translation through p70 S6K primarily promotes the translation of $5^{\prime}$ TOP $\left(5^{\prime}\right.$ terminal 
A
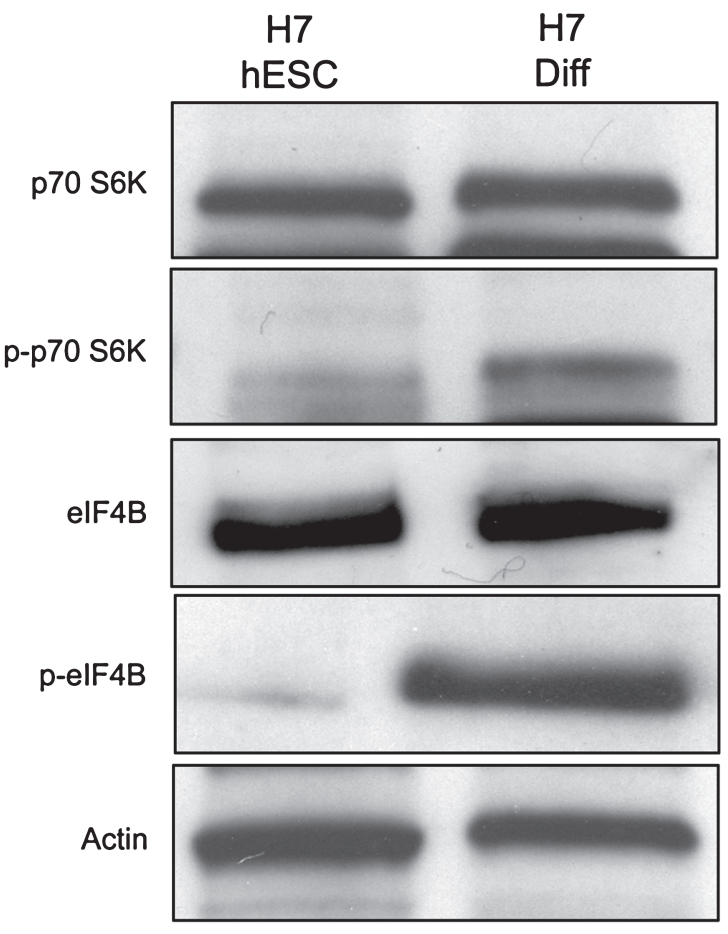

B

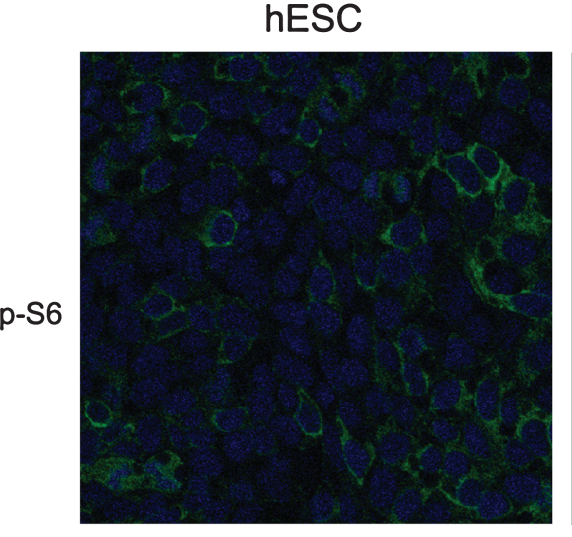

$\mathrm{H7}$

Diff

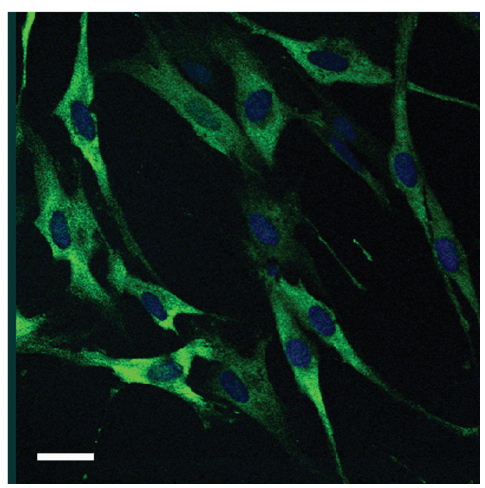

FIG. 2. H7 hESCs show reduced levels of p70 S6K signaling compared to their differentiated progeny. (A) Representative Western blots from four separate experiments indicating decreased p70 S6K activation in hESCs compared to differentiated, fibroblastlike cells. (B) Representative images showing reduced S6 phosphorylation in $\mathrm{H} 7 \mathrm{hESCs}$ compared to H7 Diffs. S6 phosphorylation appears to be increased along the outer edges of hESC colonies. Scale bar, $40 \mu \mathrm{m}$. oligopyrimidine tract) mRNAs, which encode proteins associated with protein translation (initiation factor complex proteins) and cell cycle progression (Jefferies et al., 1997). Consequently, rapamycin has been shown to suppress expression of $5^{\prime}$ TOP mRNAs (Jefferies et al., 1997). To determine the extent at which mTORC1-mediated translation contributes to global protein translation in hESCs, we cultured H7 hESCs in a feeder-free system for $24 \mathrm{~h}$ in medium containing $\left[{ }^{35} \mathrm{~S}\right]$ methionine in the presence of the following conditions: control DMSO, $0.2 \mu \mathrm{M}$ Emetine, $1 \mu \mathrm{g} / \mu \mathrm{L}$ Puromycin or $20 \mathrm{nM}$ Rapamycin. After $24 \mathrm{~h}$, cells were harvested for total protein and subjected to SDS-PAGE. Following transfer to nitrocellulose for autoradiography analysis, blots were exposed to film for 5 days at $-80^{\circ} \mathrm{C}$ to obtain a strong signal in hESCs. Emetine and Puromycin, two global translation inhibitors, blocked nearly all protein translation in $\mathrm{H7}$ hESCs (Fig. 4A). On the other hand, rapamycin treatment only slightly decreased total protein translation (Fig. 4A).
This result suggests that only a fraction of the protein translation occurring in hESCs is regulated through the mTORC1/p70 S6K pathway.

Interestingly, rapamycin treatment suppressed new synthesis of a protein that migrates on SDS-PAGE at $85 \mathrm{kDA}$ (Fig. 4A). This protein most likely corresponds to a protein translation initiation factor such as eIF4B or eIF2B $\varepsilon$, both of which also represent members of the $5^{\prime}$ TOP mRNA family. Although no change in protein expression was observed with 24-h or 3-day rapamycin treatment (Fig. 4A and B), 6-day treatment with rapamycin significantly $(p<0.01)$ decreased expression of eIF4B and eIF2B $\varepsilon$ (Fig. 4B). These results suggest that prolonged treatment with rapamycin may negatively impact global protein translation by depleting expression of $5^{\prime}$ TOP family proteins that regulate translation initiation, and may contribute to the long-term detrimental effects of rapamycin treatment on hESCs (Zhou et al., 2009). 
A

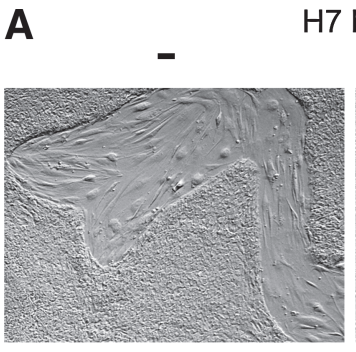

H7 hESC

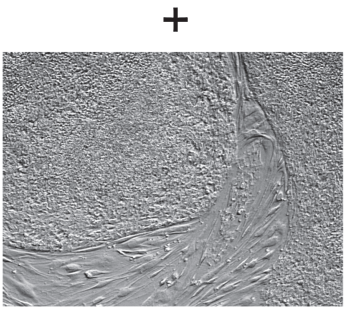

B
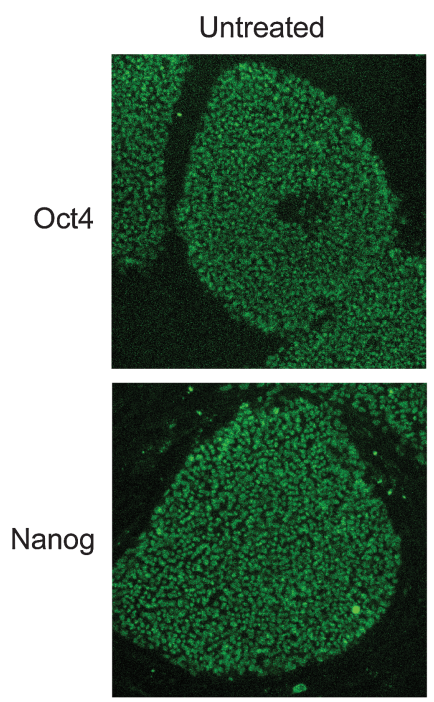

Rapamycin
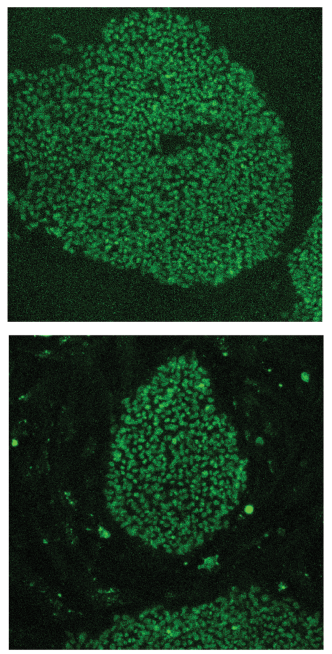

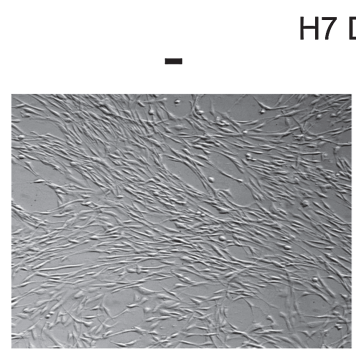

H7 Diff

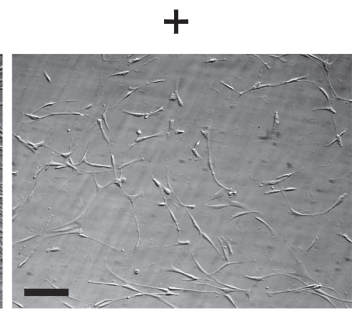

siRNA
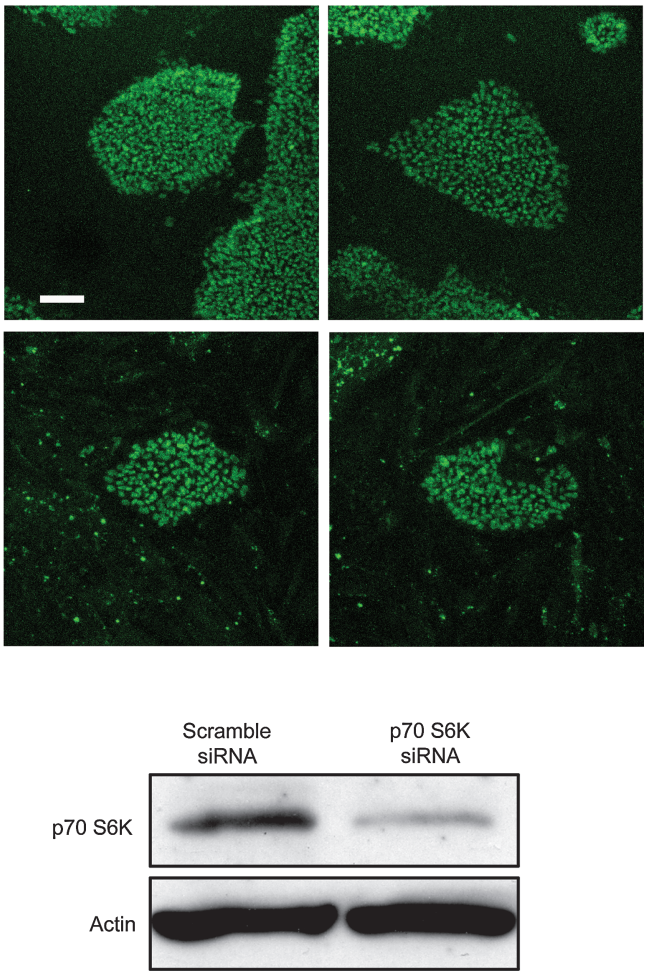

FIG. 3. Suppression of mTORC1-mediated translation does not induce cell death or disrupt pluripotency in H7 hESCs. (A) Rapamycin treatment causes cell death in differentiated, but not undifferentiated cells. Representative images of untreated and treated H7 hESCs and H7 Diffs are shown. Scale bar, $100 \mu \mathrm{m}$. (B) Neither treatment with rapamycin nor siRNA against p70 S6K impairs colony formation or affects expression of pluripotency markers in H7 hESCs. Representative images for hESCs treated for 3 days with vehicle control or $20 \mathrm{nM}$ rapamycin are shown. Also shown are representative images from H7 hESCs nucleofected with either control, nonspecific siRNA, or siRNA directed against p70 S6K. Typically, hESC colonies are smaller post nucleofection due to the requirement of single-cell suspensions. Staining for Oct4 and Nanog pluripotency markers are shown. Scale bar, $100 \mu \mathrm{M}$. Western blot insert confirms p70 S6K knockdown in hESCs with Actin serving as a loading control.

\section{Expression of constitutively active p70 S6K causes differentiation of $\mathrm{H} 7 \mathrm{hESC}$}

We next examined whether expression of either wild-type p70 S6K (p70 S6K WT) or constitutively active p70 S6K (p70 S6K CON) had any effect on the maintenance of pluripotency in hESCs. p70 S6K can be constitutively activated through a series of four point mutations within the catalytic, linker, and pseudosubstrate domains (Schalm and Blenis, 2002). Using the Amaxa Nucleofector II system (Hohenstein et al., 2008), H7 hESCs were nucleofected with GFP alone or conucleofected with GFP + p70 S6K WT or GFP + p70 S6K CON. Approximately 50-60\% nucleofection efficiency was typically observed when nucleofecting plasmid DNA (data not shown). Three days post nucleofection, cells were fixed and stained for Oct 4 . Greater than $96 \%$ of cells expressing GFP or GFP + p70 S6K WT expressed Oct4 (Fig. 5A and B). Likewise, these GFP + /Oct4 expressing cells possessed a high nuclear-tocytoplasmic ratio (Fig. 5, see arrows), suggesting that these cells retained similar characteristics to undifferentiated pluripotent stem cells. However, expression of p70 S6K CON significantly reduced $(p<0.01)$ Oct4 expression in GFP + cells (Fig. 5A and B). Furthermore, the majority of GFP + cells showed a fibroblast-like morphology with a low nuclear-to-cytoplasmic ratio (Fig. 5, see arrows). These results indicate that upregulation of p70 S6K activity induces differentiation in pluripotent hESCs. 
A
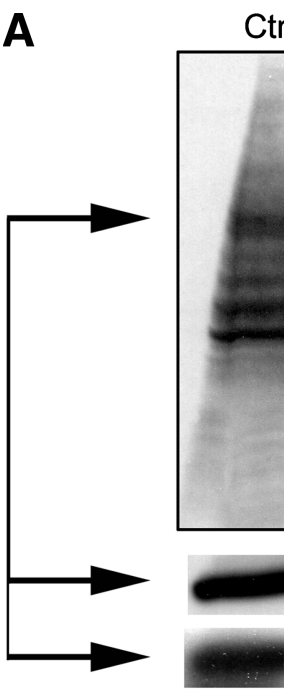

Emt

Pur

Rap

B
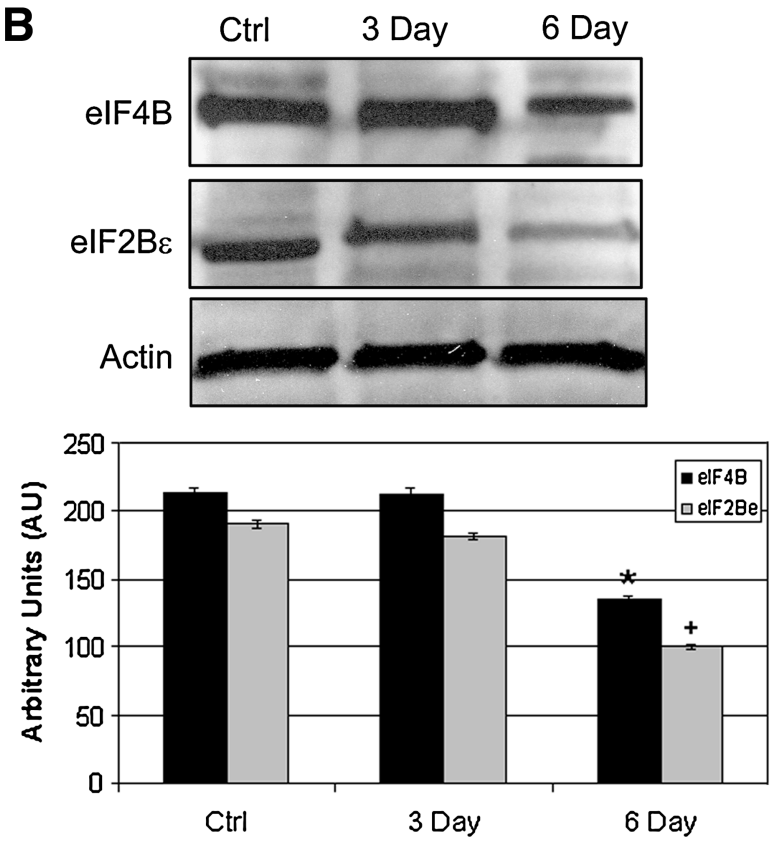

FIG. 4. Prolonged treatment with rapamycin reduces expression of eIF4B and eIF2B $\varepsilon$. (A) Rapamycin treatment slightly reduces global protein translation in H7 hESCs. Representative autoradiograph and Western blots from three separate experiments indicating that rapamycin only slightly decreases protein synthesis compared to global translation inhibitors puromycin and emetine as determined by [ $\left.{ }^{35} \mathrm{~S}\right]$ methionine incorporation. Arrow indicates an $\sim 85-\mathrm{kDA}$ band that shows reduced new synthesis in rapamycin-treated samples. Western blots for two proteins that fall within this size range, $\mathrm{eIF} 4 \mathrm{~B}$ and $\mathrm{eIF} 2 \mathrm{~B} \varepsilon$, are shown. (B) Six-day treatment of $20 \mathrm{nM}$ rapamycin significantly decreases expression of eIF4B and eIF2B $\varepsilon$. Representative Western blots from three separate experiments showing eIF4B and $\mathrm{eIF} 2 \mathrm{~B} \varepsilon$ expression in response to rapamycin treatment.

siRNA-mediated knockdown of TSC2 and Rictor reduces Oct4 expression and alters cell morphology of pluripotent $\mathrm{H} 7 \mathrm{hESCS}$

Because elevation in p70 S6K induced differentiation in hESCs, we next examined the expression of mTOR complex
A

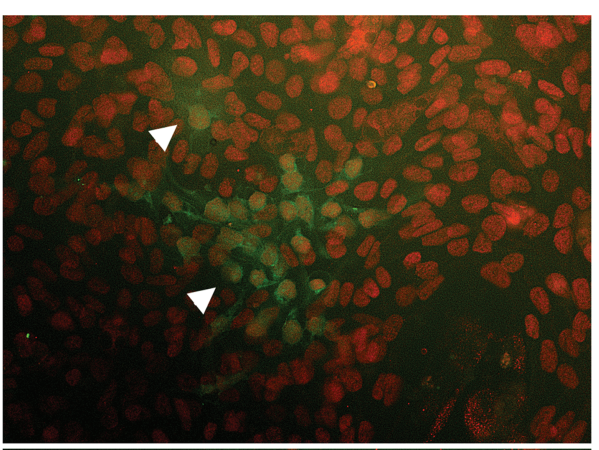

GFP + p70 S6K WT

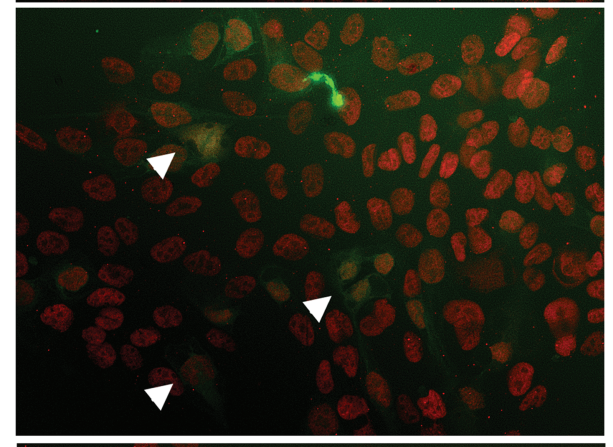

GFP + p70 S6K CON

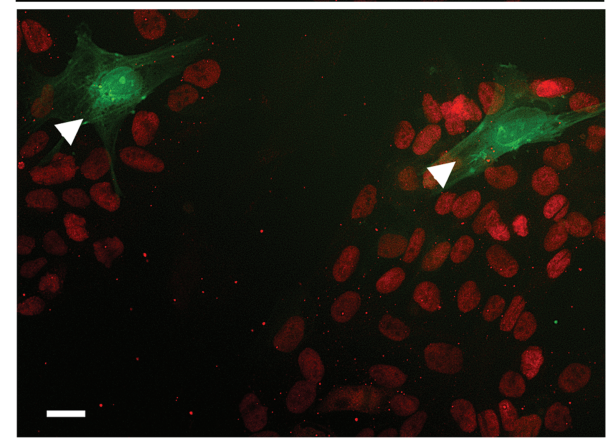

B

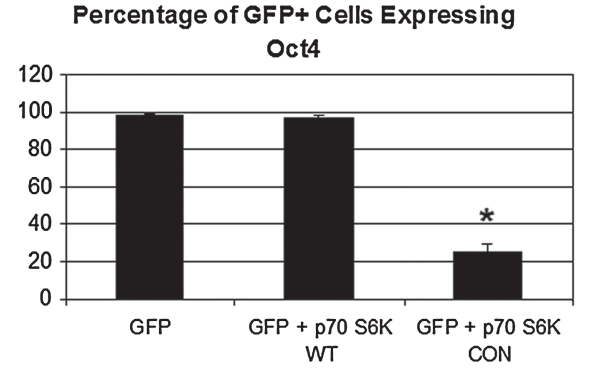

FIG. 5. Expression of constitutively active p70 S6K alters cell morphology and causes a loss of Oct4 expression in H7 hESCs. Constitutively active p70 S6K induces differentiation of H7 hESCs. (A) Representative fluorescent imaging of H7 hESC nucleofected with GFP, GFP + p70 S6K WT, or $\mathrm{GFP}+$ p70 S6K CON and stained for Oct4. Arrows indicate nucleofected cells. Scale bar, $50 \mu \mathrm{m}$. (B) Graphical analysis of the percentage of GFP + cells expressing Oct4. For each condition, approximately 300 cells were analyzed. Statistical significance, $p<0.01$.

proteins and mTOR regulatory proteins in $\mathrm{H} 7 \mathrm{hESCs}$ and H7 Diffs.. H7 hESCs were cultured in a feeder-free system and harvested 6 days post passaging on Matrige ${ }^{\mathrm{TM}}$ dishes. Similarly, H7 Diffs were harvested at $70-80 \%$ confluency. Following harvesting, H7 hESCs and H7 Diffs were 
subjected to SDS-PAGE followed by Western blot analysis for the proteins indicated. H7 hESCs express higher levels of the mTORC2 binding protein Rictor and the mTORC1 inhibitory protein TSC2 compared to their differentiated progeny (Fig. 6A). Likewise, H7 hESCs exhibit higher levels of the lower doublet band for TSC1 compared to H7 Diffs. This band represents the dephosphorylated, active form of TSC1, which promotes binding to TSC2 to inhibit Rhebmediated mTORC1 activation (Huang and Manning, 2008).

To identify mTORC composition in hESCs and their differentiated progeny, we immunoprecipitated mTOR from cell lysates and probed for either Raptor (mTORC1 binding protein) or Rictor (mTORC2 binding protein). H7 hESCs preferentially express more mTORC2 than mTORC1, whereas H7 Diffs exhibit higher expression of mTORC1 compared to mTORC2 (Fig. 6B). As previously mentioned, TSC1 and TSC2 association represents a potent negative regulator of mTORC1 signaling (Huang and Manning, 2008). To determine whether this complex is active in hESCs, we immunoprecipitated TSC1 from cell lysates of H7 hESCs and H7 Diffs and probed for TSC2. H7 hESCs expressed significantly higher levels of the TSC1/TSC2 inhibitory complex compared to their differentiated progeny (Fig. 6B). To further demonstrate that restricting mTORC1 signaling is critical for the maintenance of pluripotency in hESCs, we introduced siRNA directed at TSC2 and Rictor in an attempt to upregulate mTORC1 activity using the Amaxa Nucleofector II system (Hohenstein et al., 2008). H7 hESCs nucleofected with either TSC2 or Rictor siRNA alone showed no change in colony formation, Oct4 expression, or increased p70 S6K activation, although hESC colonies were slightly smaller in size with Rictor siRNA (data not shown). Instead, H7 hESCs nucleofected with both TSC2 and Rictor siRNAs exhibited changes in cell morphology, which included a flatter cell appearance and a higher cytoplasm to nuclear ration, a slight increase in p70 S6K activation, and a concomitant decrease in Oct4 expression compared to hESCs nucleofected with control, nonspecific siRNA (Fig. 6C and D). These results suggest that mTORC1 signaling is restricted in hESCs compared to their differentiated progeny, and that elevations in mTORC1 activity alter stem cell pluripotency.

\section{Discussion}

The results from this study demonstrate a unique role for mTORC1/p70 S6K-mediated protein translation in inducing hESC differentiation. Previous work in mESCs and differentiated cells has shown that global translation rates are reduced in embryonic stem cells (Sampath et al., 2008). Here, we show that endogenous levels of activated p70 S6K are lower in pluripotent hESCs compared to differentiated fibroblast-like cells, whereas the expression of TSC1/TSC2mTORC1 inhibitory complex is higher in hESCs. Furthermore, we show that inhibition of p70 S6K signaling through
FIG. 6. Knockdown of Rictor and TSC2 expression elevates p70 S6K activation and alters hESC morphology. Activation of p70 S6K by Rictor and TSC2 siRNA-mediated knockdown leads to changes in cell morphology to a more differentiated phenotype. (A) Representative Western blots from multiple experiments showing expression of proteins associated with mTORC1 (mammalian target of rapamycin complex 1) and mTORC2 signaling in $\mathrm{H} 7$ hESCs and H7 Diffs. (B) Western blot of immunoprecipitations of mTOR or TSC1 probed for Raptor, Rictor, or TSC2, respectively, in H7 hESCs and H7 Diffs. (C) Representative phase contrast images of $\mathrm{H} 7$ hESCs nucleofected with either control siRNA or Rictor/TSC2 siRNA mixture. Scale bar, $250 \mu \mathrm{m}$. (D) Western blot analysis confirming knockdown of TSC2 and Rictor as well as expression of p70 S6K, activated p70 S6K (p-T389), Oct4, and Actin.
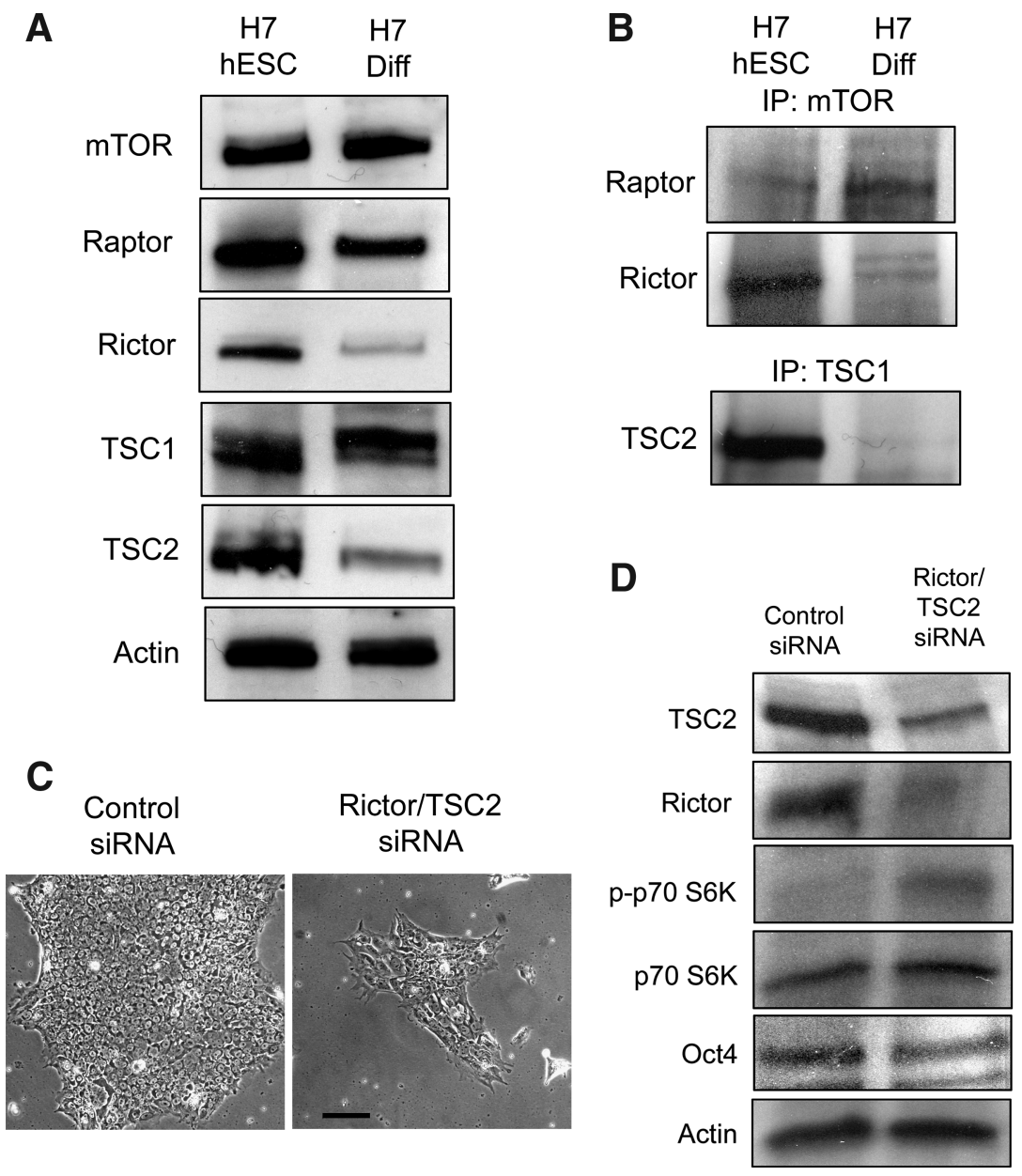


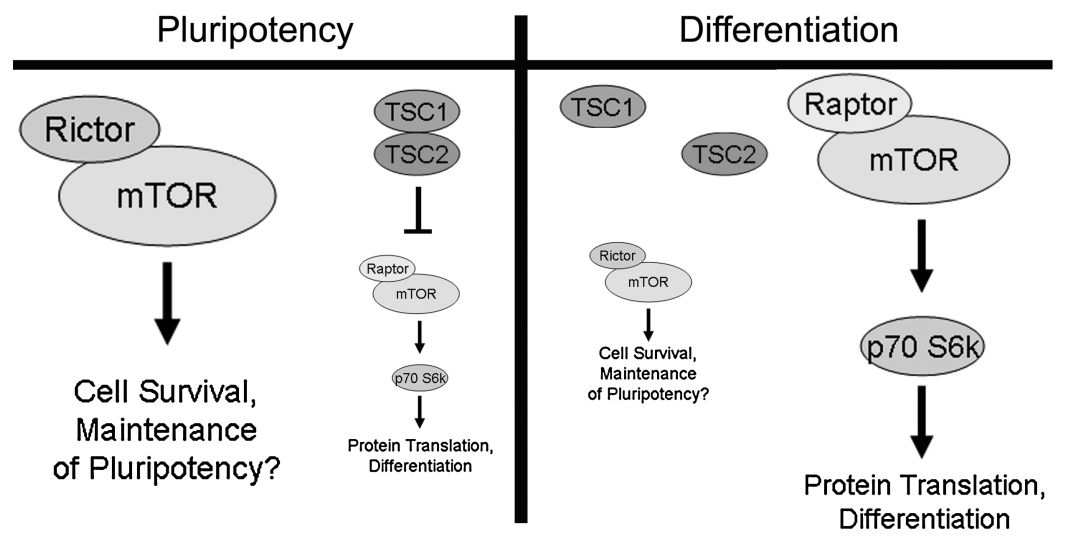

FIG. 7. Diagram depicting mTORC signaling in hESCs and differentiated cells. In pluripotent hESCs (left panel), mTOR protein is mostly associated in mTORC2 (mTORRictor), whereas mTORC1 (mTOR-Raptor) activity is suppressed by the heterodimer TSC1/TSC2. In differentiated cells (right panel), mTOR is highly associated with Raptor over Rictor leading to elevated p70 S6K activity. Increased protein translation, in turn, induces differentiation. either rapamycin treatment or siRNA-mediated knockdown has no effect on the maintenance of pluripotency in hESCs over 3 days. Conversely, expression of constitutively active p70 S6K, but not wild-type p70 S6K, induces differentiation of hESCs. Likewise, knockdown of Rictor and TSC2 stimulates p70 S6K activation, decreases Oct4 expression, and alters cell morphology of hESCs. These results implicate that tight regulation of $\mathrm{mTORC} 1$ signaling is required for the maintenance of pluripotency in hESCs (Fig. 7).

The activation of p70 S6K resulting in an increase in protein synthesis is proposed as a physiological regulator in the cascade driving a pluripotent stem cell into differentiation. The onset of protein synthesis has been found in many developmental models as vital for generating and maintaining a differentiated state. Specifically, primordial germ cells are maintained in a pluripotent state through suppression of global transcription and translation (Santos and Lehmann, 2004; Strome and Lehmann, 2007). Here, we suggest that p70 $\mathrm{S} 6 \mathrm{~K}$ is among the natural activators of translation, and not adventitiously triggering translation, because its effect is limited only with pluripotent, not differentiated cells. Interestingly, blocking global translation with 24-36-h emetine or puromycin treatment causes cell death in hESCs (data not shown). These results suggest that the low levels of translation observed in hESCs are vital to pluripotent stem cell survival. Investigation into the differential protein expression in hESCs treated with emetine, puromycin, and rapamycin may lead to the delineation of a protein network critical for maintaining or inducing pluripotency.

Procedures for inducing pluripotency are being reported rapidly, and preventing the increase in translation accompanying differentiation may prove helpful for inducing or maintaining pluripotency. Several researchers have shown that viral infection of defined transcriptional regulators initiates reprogramming of adult somatic cells into pluripotent stem-like cells (Aoi et al., 2008; Liu et al., 2008; Lowry et al., 2008; Maherali et al., 2007; Meissner et al., 2007; Nakagawa et al., 2008; Park et al., 2008; Takahashi et al., 2007; Wernig et al., 2007, 2008; Yu et al., 2007). Although this groundbreaking research has revolutionized how stem-like cells can be obtained in culture, this work is still limited in direct clinical relevance, as many reprogramming protocols require viral integration of transcription factors, some of which represent oncogenes (i.e., c-myc, Klf4, and Lin-28). Although some researchers have utilized adenoviral vectors, miRNA, or a combination of genetic and chemical treatment (Li et al.,
2009; Stadtfeld et al., 2008; Varas et al., 2009), chemically inducing pluripotency represents the most clinically relevant solution. As such, suppression of mTORC1-mediated translation may perhaps be another strategy for the chemical induction of pluripotency. Because rapamycin does not affect cell viability in pluripotent stem cells, targeting mTOR or p70 S6K-mediated translation may also prove useful as a selection agent following the induction of pluripotency. Thus, discovery of the downstream modulators responsible for increases in global translation rates is a worthwhile future endeavor for understanding the mechanisms whereby suppression of p70 S6K-mediated translation maintains pluripotency.

Recently, Zhou et al. (2009) showed that prolonged rapamycin treatment induces differentiation of hESCs through a loss of pluripotency markers and changes in cell morphology. Our results suggest that a loss of Oct4 and Nanog may be an off-target result from a rapamycin-induced reduction in expression of protein translational machinery. Zhou et al. (2009) also demonstrated that a knockout of mTOR induced rapid differentiation of hESCs. This result could be due to a reduction in both $\mathrm{mTORC} 1$ and mTORC2 activity. Furthermore, prolonged rapamycin treatment has been shown to disrupt mTORC2 assembly and limit mTORC2 activity in certain cell types (Sarbassov et al., 2006). Thus, mTORC2 actually may serve as the primary complex for maintaining hESCs in their pluripotent state rather than mTORC1, especially considering the overabundance of mTORC2 in hESCs compared to their differentiated progeny (Fig. 7).

A recent study by Harrison et al. (2009) indicates that rapamycin treatment extends the life span of mice. This innovative research demonstrates a critical role for mTORC1 signaling in the onset of organismal aging. One potential theory exists that suggests that stem cell aging contributes to organismal aging (Blagosklonny, 2008). Prolonged/ hyperactive mTORC1 signaling has been shown to block numerous signaling pathways, including insulin and PDGF signaling (for review, see Blagosklonny, 2008). Desensitizing stem cells to these pathways may contribute to differentiation, cellular aging, etc. Brief treatment with rapamycin may "clear the mTORC1 signaling slate" and resensitize stem cells to certain signaling pathways. Likewise, this cellular rejuvenation may contribute to the increased lifespan seen in rapamycin-fed mice by preventing stem cell aging. For embryonic stem cells, strict regulation of mTORC1 appears necessary for maintaining pluripotency, whereas short-term rapamycin treatment may serve to improve colony growth. 


\section{Acknowledgments}

The authors thank Dr. Kyle Orwig for his assistance with the radioactive protein translation experiments, Dr. Chris Navara for constructive discussions, and Helen Fong and Ellen Smith from Dr. Peter Donovan's Lab for their technical assistance with stem cell nucleofection. This work was supported by grant P01 HD 47675 from the National Institutes of Health.

\section{Author Disclosure Statement}

The authors declare that no conflicting financial interests exist.

\section{References}

Alessi, D.R., Kozlowski, M.T., Weng, Q.P., et al. (1998). 3-Phosphoinositide-dependent protein kinase 1 (PDK1) phosphorylates and activates the p70 S6 kinase in vivo and in vitro. Curr. Biol. 8, 69-81.

Aoi, T., Yae, K., Nakagawa, M., et al. (2008). Generation of pluripotent stem cells from adult mouse liver and stomach cells. Science 321, 699-702.

Blagosklonny, M.V. (2008). Aging, stem cells, and mammalian target of rapamycin: a prospect of pharmacologic rejuvenation of aging stem cells. Rejuvenat. Res. 11, 801-808.

Chang, W.Y., and Stanford, W.L. (2008). Translational control: a new dimension in embryonic stem cell network analysis. Cell Stem Cell 2, 410-412.

Dennis, P.B., Jaeschke, A., Saitoh, M., et al. (2001). Mammalian TOR: a homeostatic ATP sensor. Science 294, 1102-1105.

Dufner, A., and Thomas, G. (1999). Ribosomal S6 kinase signaling and the control of translation. Exp. Cell Res. 253, 100-109.

Dvash, T., Mayshar, Y., Darr, H., et al. (2004). Temporal gene expression during differentiation of human embryonic stem cells and embryoid bodies. Hum. Reprod. 19, 2875-2883.

Easley, C.A., IV., Brown, C.M., Horwitz, A.F., et al. (2008). CaMK-II promotes focal adhesion turnover and cell motility by inducing tyrosine dephosphorylation of FAK and paxillin. Cell Motil. Cytoskeleton 65, 662-674.

Fang, Y., Vilella-Bach, M., Bachmann, R., et al. (2001). Phosphatidic acid-mediated mitogenic activation of mTOR signaling. Science 294, 1942-1945.

Gingras, A.C., Raught, B., Gygi, S.P., et al. (2001a). Hierarchical phosphorylation of the translation inhibitor 4E-BP1. Genes Dev. 15, 2852-2864.

Gingras, A.C., Raught, B., and Sonenberg, N. (2001b). Regulation of translation initiation by FRAP/mTOR. Genes Dev. 15, 807-826.

Gunji, W., Kai, T., Sameshima, E., et al. (2004). Global analysis of the expression patterns of transcriptional regulatory factors in formation of embryoid bodies using sensitive oligonucleotide microarray systems. Biochem. Biophys. Res. Commun. 325, 265-275.

Harrison, D.E., Strong, R., Sharp, Z.D., et al. (2009). Rapamycin fed late in life extends lifespan in genetically heterogeneous mice. Nature 460, 392-395.

Hohenstein, K.A., Pyle, A.D., Chern, J.Y., et al. (2008). Nucleofection mediates high-efficiency stable gene knockdown and transgene expression in human embryonic stem cells. Stem Cells 26, 1436-1443.

Holz, M.K., and Blenis, J. (2005). Identification of S6 kinase 1 as a novel mammalian target of rapamycin (mTOR)-phosphorylating kinase. J. Biol. Chem. 280, 26089-26093.
Huang, J., and Manning, B.D. (2008). The TSC1-TSC2 complex: a molecular switchboard controlling cell growth. Biochem. J. 412, 179-190.

Ivanova, N.B., Dimos, J.T., Schaniel, C., et al. (2002). A stem cell molecular signature. Science 298, 601-604.

Ivanova, N., Dobrin, R., Lu, R., et al. (2006). Dissecting selfrenewal in stem cells with RNA interference. Nature 442, 533-538.

Jacinto, E., Loewith, R., Schmidt, A., et al. (2004). Mammalian TOR complex 2 controls the actin cytoskeleton and is rapamycin insensitive. Nat. Cell Biol. 6, 1122-1128.

Jefferies, H.B., Fumagalli, S., Dennis, P.B., et al. (1997). Rapamycin suppresses 5'TOP mRNA translation through inhibition of p70s6k. EMBO J. 16, 3693-3704.

Kawasome, H., Papst, P., Webb, S., et al. (1998). Targeted disruption of p70(s6k) defines its role in protein synthesis and rapamycin sensitivity. Proc. Natl. Acad. Sci. USA 95, 50335038.

Lee, T.I., Jenner, R.G., Boyer, L.A., et al. (2006). Control of developmental regulators by Polycomb in human embryonic stem cells. Cell 125, 301-313.

Li, W., Wei, W., Zhu, S., et al. (2009). Generation of rat and human induced pluripotent stem cells by combining genetic reprogramming and chemical inhibitors. Cell Stem Cell 4: 359-369.

Liu, H., Zhu, F., Yong, J., et al. (2008). Generation of induced pluripotent stem cells from adult rhesus monkey fibroblasts. Cell Stem Cell 3, 587-590.

Loewith, R., Jacinto, E., Wullschleger, S., et al. (2002). Two TOR complexes, only one of which is rapamycin sensitive, have distinct roles in cell growth control. Mol. Cell 10, 457-468.

Lowry, W.E., Richter, L., Yachechko, R., et al. (2008). Generation of human induced pluripotent stem cells from dermal fibroblasts. Proc. Natl. Acad. Sci. USA 105, 2883-2888.

Ludwig, T.E., Bergendahl, V., Levenstein, M.E., et al. (2006a). Feeder-independent culture of human embryonic stem cells. Nat. Methods 3, 637-646.

Ludwig, T.E., Levenstein, M.E., Jones, J.M., et al. (2006b). Derivation of human embryonic stem cells in defined conditions. Nat. Biotechnol. 24, 185-187.

Maherali, N., Sridharan, R., Xie, W., et al. (2007). Directly reprogrammed fibroblasts show global epigenetic remodeling and widespread tissue contribution. Cell Stem Cell 1, 55-70.

Meissner, A., Wernig, M., and Jaenisch, R. (2007). Direct reprogramming of genetically unmodified fibroblasts into pluripotent stem cells. Nat. Biotechnol. 25, 1177-1181.

Nakagawa, M., Koyanagi, M., Tanabe, K., et al. (2008). Generation of induced pluripotent stem cells without Myc from mouse and human fibroblasts. Nat. Biotechnol. 26, 101-106.

Navara, C.S., Mich-Basso, J.D., Redinger, C.J., et al. (2007a). Pedigreed primate embryonic stem cells express homogeneous familial gene profiles. Stem Cells 25, 2695-2704.

Navara, C.S., Redinger, C., Mich-Basso, J., et al. (2007b). Derivation and characterization of nonhuman primate embryonic stem cells. Curr. Protoc. Stem Cell Biol. Chapter 1, Unit 1A 1.

Park, I.H., Zhao, R., West, J.A., et al. (2008). Reprogramming of human somatic cells to pluripotency with defined factors. Nature 451, 141-146.

Pritsker, M., Ford, N.R., Jenq, H.T., et al. (2006). Genomewide gain-of-function genetic screen identifies functionally active genes in mouse embryonic stem cells. Proc. Natl. Acad. Sci. USA 103, 6946-6951.

Pullen, N., and Thomas, G. (1997). The modular phosphorylation and activation of p70s6k. FEBS Lett. 410, 78-82. 
Pullen, N., Dennis, P.B., Andjelkovic, M., et al. (1998). Phosphorylation and activation of p70s6k by PDK1. Science 279, 707-710.

Ramalho-Santos, M., Yoon, S., Matsuzaki, Y., et al. (2002). "Stemness": transcriptional profiling of embryonic and adult stem cells. Science 298, 597-600.

Raught, B., Gingras, A.C., and Sonenberg, N. (2001). The target of rapamycin (TOR) proteins. Proc. Natl. Acad. Sci. USA 98, 7037-7044

Sabers, C.J., Martin, M.M., Brunn, G.J., et al. (1995). Isolation of a protein target of the FKBP12-rapamycin complex in mammalian cells. J. Biol. Chem. 270, 815-822.

Sampath, P., Pritchard, D.K., Pabon, L., et al. (2008). A hierarchical network controls protein translation during murine embryonic stem cell self-renewal and differentiation. Cell Stem Cell 2, 448-460.

Santos, A.C., and Lehmann, R. (2004). Germ cell specification and migration in Drosophila and beyond. Curr. Biol. 14, R578-R589.

Sarbassov, D.D., Ali, S.M., Sengupta, S., et al. (2006). Prolonged rapamycin treatment inhibits mTORC2 assembly and Akt/ PKB. Mol Cell 22, 159-168.

Schalm, S.S., and Blenis, J. (2002). Identification of a conserved motif required for mTOR signaling. Curr. Biol. 12, 632-639.

Stadtfeld, M., Nagaya, M., Utikal, J., et al. (2008). Induced pluripotent stem cells generated without viral integration. Science $322,945-949$.

Strome, S., and Lehmann, R. (2007). Germ versus soma decisions: lessons from flies and worms. Science 316, 392-393.

Takahashi, K., Tanabe, K., Ohnuki, M., et al. (2007). Induction of pluripotent stem cells from adult human fibroblasts by defined factors. Cell 131, 861-872.

Varas, F., Stadtfeld, M., De Andres-Aguayo, L., et al. (2009). Fibroblast derived induced pluripotent stem cells show no common retroviral vector insertions. Stem Cells 27:300-306.
Walker, E., Ohishi, M., Davey, R.E., et al. (2007). Prediction and testing of novel transcriptional networks regulating embryonic stem cell self-renewal and commitment. Cell Stem Cell 1, 71-86.

Weng, Q.P., Kozlowski, M., Belham, C., et al. (1998). Regulation of the p70 S6 kinase by phosphorylation in vivo. Analysis using site-specific anti-phosphopeptide antibodies. J. Biol. Chem. 273, 16621-16629.

Wernig, M., Meissner, A., Foreman, R., et al. (2007). In vitro reprogramming of fibroblasts into a pluripotent ES-cell-like state. Nature 448, 318-324.

Wernig, M., Meissner, A., Cassady, J.P., et al. (2008). c-Myc is dispensable for direct reprogramming of mouse fibroblasts. Cell Stem Cell 2, 10-12.

Wiederrecht, G.J., Sabers, C.J., Brunn, G.J., et al. (1995). Mechanism of action of rapamycin: new insights into the regulation of G1-phase progression in eukaryotic cells. Prog. Cell Cycle Res. 1, 53-71.

Yu, J., Vodyanik, M.A., Smuga-Otto, K., et al. (2007). Induced pluripotent stem cell lines derived from human somatic cells. Science 318, 1917-1920.

Zhou, J., Su, P., Wang, L., et al. (2009). mTOR supports longterm self-renewal and suppresses mesoderm and endoderm activities of human embryonic stem cells. Proc. Natl. Acad. Sci. USA 106, 7840-7845.

Address correspondence to: Dr. Gerald P. Schatten

Ob/Gyn, Reproductive Sciences and Cell Biology E Physiology University of Pittsburgh 204 Craft Ave, B630 Pittsburgh, PA 15213

E-mail: schattengp@upmc.edu 
